\title{
Glioblastomas brain tumour segmentation based on convolutional neural networks
}

\author{
Moh'd Rasoul Al-Hadidi', Bayan AlSaaidah², Mohammed Y. Al-Gawagzeh ${ }^{3}$ \\ ${ }^{1,3}$ Department of Electrical Power Engineering, Department of Computer Engineering, Faculty of Engineering, \\ Al-Balqa Applied University, Jordan \\ ${ }^{2}$ Department of Computer Science, Prince Abdullah bin Ghazi Faculty of Information Technology and Communications, \\ Al-Balqa Applied University, Jordan
}

\begin{tabular}{l} 
Article Info \\
\hline Article history: \\
Received Oct 15, 2019 \\
Revised Mar 14, 2020 \\
Accepted Mar 25, 2020 \\
\hline
\end{tabular}

\section{Keywords:}

Brain tumour

Convolutional neural networks superpixel

Image segmentation

Pixel clustering

\begin{abstract}
Brain tumour segmentation can improve diagnostics efficiency, rise the prediction rate and treatment planning. This will help the doctors and experts in their work. Where many types of brain tumour may be classified easily, the gliomas tumour is challenging to be segmented because of the diffusion between the tumour and the surrounding edema. Another important challenge with this type of brain tumour is that the tumour may grow anywhere in the brain with different shape and size. Brain cancer presents one of the most famous diseases over the world, which encourage the researchers to find a high-throughput system for tumour detection and classification. Several approaches have been proposed to design automatic detection and classification systems. This paper presents an integrated framework to segment the gliomas brain tumour automatically using pixel clustering for the MRI images foreground and background and classify its type based on deep learning mechanism, which is the convolutional neural network. In this work, a novel segmentation and classification system is proposed to detect the tumour cells and classify the brain image if it is healthy or not. After collecting data for healthy and non-healthy brain images, satisfactory results are found and registered using computer vision approaches. This approach can be used as a part of a bigger diagnosis system for breast tumour detection and manipulation.
\end{abstract}

Copyright $@ 2020$ Institute of Advanced Engineering and Science. All rights reserved.

\section{Corresponding Author:}

Moh'd Rasoul Al-Hadidi,

Department of Electrical Power Engineering, Department of Computer Engineering,

Faculty of Engineering, Al-Balqa Applied University,

King Talal Street, Salt 19117, Jordan.

Email: mohammad_hadidi@bau.edu.jo

\section{INTRODUCTION}

Brain cancer present one of the highest death causes besides several cancer types with the highest death compared with the number of patients [1]. The brain tumour is a group of abnormal cells that grow in the brain [2]. Detect this mass and identify the location of it helps the doctors to treat the patients; in most cases, they need to remove the tumour surgically. Where the brain tumour has many types, gliomas present the most difficult one for prediction.

In the gliomas type, the tumour area poorly contrasts and difficult to segment regarding its diffusing. Furthermore, the tumour spread in many size and shapes in the brain [3]. In spite of the last improvement in the brain cancer treatment that happened recently, but the morbidity still correlated with the poor diagnosis. According to the American Brain tumour Association states, there are 120 types of the brain tumour and it becomes the most death cause of the young people whose age under 40 years [4]. Despite all the improvements in the brain cancer treatment but the survival rate still low, which as reported in the cure brain cancer foundation and shown in Figure 1 [5]. 
Early detection of brain cancer can help the patient to be survived and overcome cancer treatment problems. The low survival percent, the high cost of the treatment, the severity behind the surgery treatment, and a large number of brain types present demand for early detection with an effective diagnosis. The most popular imaging method for medical purposes is the magnetic resonance imaging (MRI) method [6] in which a strong magnetic field is used besides the radio waves and the field gradients. Depending on the clinical application, different types of contrast that are used in MR imaging like T1-and T2-weighted imaging [7]. An example of the MRI images is shown in Figure 2.

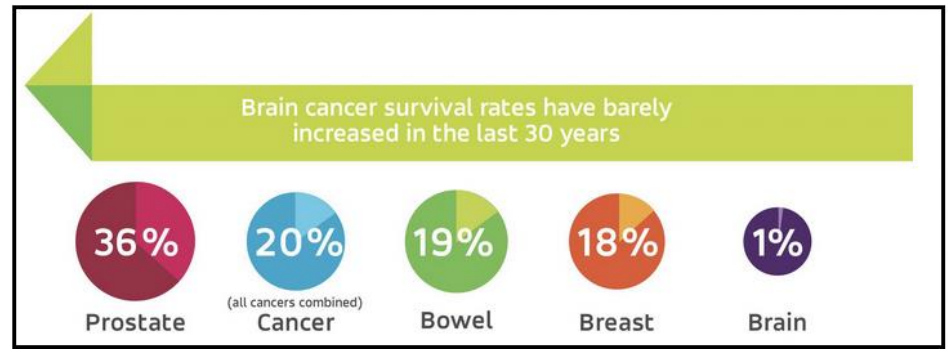

Figure 1. Survival rate for the period between 1984-2013 [5]

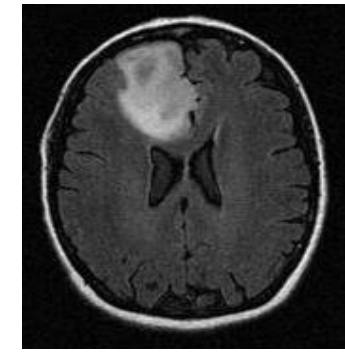

Figure 2. Brain image using MRI technique

Classifying the brain cells if it is healthy or not, the tumour cells should be segmented first. The most popular segmentation method is a region growing method which depends on a seed point that is growing according to the Euclidian distance between pixels [8]. However, the segmentation process is considered as a challenge for researchers because of the image uniformity and the variation of the cells size and shape [9].

Superpixel method is a simple type of clustering that is used for image partitioning process [10] and based on the most important part of any image which is the pixel value [11]. Using several parameters and depending on the distance between pixels, these partitions are segmented and labelled with variant sizes. These sub-images are used as input for classification models for classification purposes.

Convolutional neural network $(\mathrm{CNN})$ is considered as a robust classification model that is trained and learned on a huge number of data sets and designed using a combination of networks as layers. Using the CNN means the ability to extract features from the raw input data using its complicated hierarchy without need for the manual feature extraction [12]. This study aims to segment the gliomas brain tumour automatically using pixel clustering for the MRI images foreground and background and use the results to classify the cell status based on deep learning mechanism which is the CNN.

\section{RELATED WORK}

Segmentation the brain tumour process is still a challenge for the researchers and the most common method for brain tumour segmentation is the region growing method [13]. The segmentation process using region growing need for a manual selection for a seed in which the selected point may cause an intensity distance error in the homogeneity of the of pixels. Another method may be the thresholding [14] depending on two grey levels ( 0 and 255) this may cause losing some of the actual tumour cells. Based on image processing techniques and using ANNs, the cancer cells were detected and classified [15]. This work is inspired to merge a compatiple techniques to get the most useful information from the images based on the ROI using image processing techniques.

Depending on the symmetrical points of the left and the right sides of the brain, some methods were proposed. Extract the features along the line between the two sides where low symmetry means there is different tissue which means tumour existing $[16,17]$. But this way cannot be efficient with gliomas tumour type because this type appears in some cases in various locations with different shape and size.

Using the convolutional networks in classification able to extract sophisticated features which makes them well-meaning. This is done by providing the output feature maps of a Convolutional layer as input channels to the subsequent Convolutional layer [18]. The building blocks in CNN allow forming different types of CNNs. This type of deep learning networks is very effective for high-performance computer vision model, and they efficiently learn and extract many visual features for well generalizing tasks without the need for hand-crafted feature extraction [19]. Most of the existed methods are based on clustering algorithms, machine learning, or using the whole image based on deep learning algorithms [20-23]. 
The performance of these methods depends on the quality and the type of the extracted features which can be varied [15, 24].

The main aim of this paper is to develop an integrated clustring and deep learning based approach to detect and extract the brain tumour and classify its type. Based on superpixel clustring algorithm for tumour segmentation is expected to work properly without needing for the manual detection of the tumour cells. Moreover, using the deep learning for classification purposes will be independent from the feature extraction process which is traditionally used in machine learning. Furthermore, the proposed approach showed promising results which prove the ability of the deep learning algorithm to produce a robust and accurate detection and classification system for the gliomas brain tumour.

\section{EXPERIMENT AND RESULTS}

The proposed study aims to segment the brain tumour using a superpixel clustering method then classify the labelled patches using CNN. This work was carried out over five months and will be improved subsequently for better results.

\subsection{Material and data set}

The proposed algorithm was carried out and tested using a data set from the cancer imaging archive (TCIA) $[25,26]$. This data set is publicly available and can be used for research and academic purposes. The neuroradiologists in Thomas Jefferson University (TJU) Hospital provide the image by its feature characterisations. The total number of images in this data set is 4069; the healthy brain is presented by 988 images where the non-healthy brain is presented by 3081 images.

\subsection{Experiment}

The proposed system consists of multiple stages as shown in Figure 3.

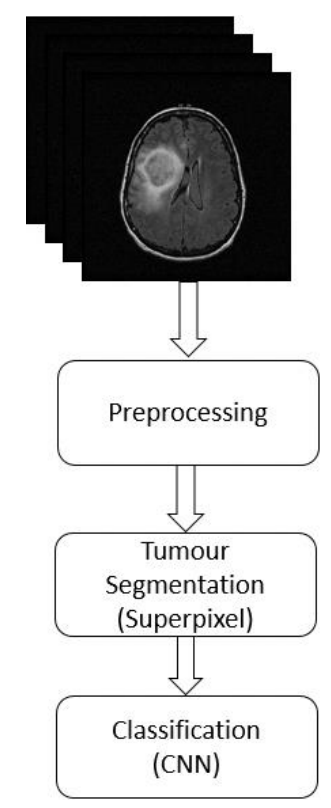

Figure 3. General methodology

\subsection{Pre-processing}

This step aims to prepare the images and adjust their contrast using filtering and normalise the images using statistical operations bbased on the following equation [27]. This step was applied to all images before the superpixel segmentation process.

$$
C=\frac{L_{\max }-L_{\min }}{L_{\max }+L_{\min }}
$$

where $C$ is the contrast, $L_{\max }$ and $L_{\min }$ are the maximum and minimum luminance values. 


\subsection{Superpixel segmentation}

After preparing the MRI images and remove any noise may appear and cause segmentation or classification error, a superpixel segmentation process was applied to segment the brain tumour area. There are different algorithms can be used for superpixel segmentation [28]. The proposed method used simple linear iterative clustering (SLIC) algorithm [10], which adaptively refines the compactness parameter after the first iteration. The first step of this algorithm is initialising centers for clusters on a grid spaced $\mathrm{S}$ pixel. Next, the cluster centers are altered into $3 \times 3$ neighborhood based on the lowest gradient position. Each pixel is assigned to the nearest pixel based on the measured distance as shown in (2) which is measured using (3) and (4) which find the color nearness and the spatial nearness respectively.

$$
\begin{aligned}
& D=\sqrt{\left(\frac{d_{c}}{m}\right)^{2}+\left(\frac{d_{s}}{s}\right)^{2}} \\
& d_{c}=\sqrt{\sum_{s_{p} \in B}\left(I\left(x_{i}, y_{i}, s_{p}\right)-I\left(x_{j}, y_{j}, s_{p}\right)\right)^{2}} \\
& d_{s}=\sqrt{\left(x_{j}-x_{i}\right)^{2}+\left(y_{j}-y_{i}\right)^{2}}
\end{aligned}
$$

$S_{p}$ is the spectral band that has the pixels $I\left(x_{i}, y_{i}, s_{p}\right)$ and $I\left(x_{j}, y_{j}, s_{p}\right), \mathrm{m}$ parameter is used to control the superpixels compactness, B presents the spectral band set. Finally, S presents the sampling interval of each cluster centroid [29].

Split the image into labels after several attempts to find the most suitable value of the number of superpixels we want to create, which is 15 areas. After computing, the number of superpixels, which is 16 , the colour of each pixel was set using the mean value of the superpixel region. This grouping process is done depending on the spatial distance and also the intensity distance between the pixels. Figure 4 shows these superpixels where Figure 5 shows the labelled regions after setting the pixel values.

Applying these steps and binarize the resultant image, the required segmented image for the non-healthy cells is produced. It is shown in Figure 6. The segmented images will be used in CNN for training purposes to predict the status of the brain cells.

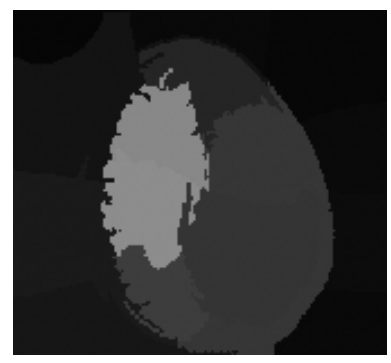

Figure 4. Pixel values setting

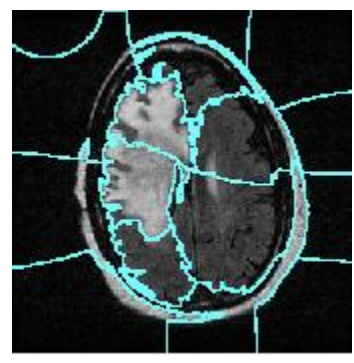

Figure 5. Image superpixels

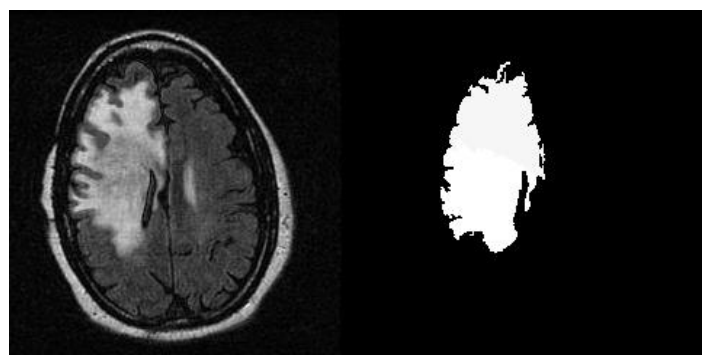

Figure 6. Segmented image

\subsection{Convolutional neural networks}

In this stage, the resulted patches or the sub-areas from the superpixel segmentation step are labelled then trained using the CNN to classify the brain cells normality. The traditional way for classification always carried out by extracting the features manually then use one of the machine learning classifiers such as neural networks and SVM. By using the deep learning network, which is CNN, significant features will be extracted using the raw images which are here the resulted patches from the superpixel step. The CNN structure comprises of many layers: the input layer, the convolutional layers, pooling layers, dropout layers, fully connected layers, and finally the output layer. These layers are explained below as shown in Figure 7.

a. Convolutional layer: This is the first layer that deals with the raw image. This layer consists of many filters that are convolved to have weights for each region of the image that is presented as a feature map [30].

b. Pooling layer: After having a huge number of features, these features are reduced using the pooling layer that will reduce the computational complexity of the network [32]. 
c. Fully connected layer: This the last layer where each neuron in this layer is connected with all neurons in the previous layer.

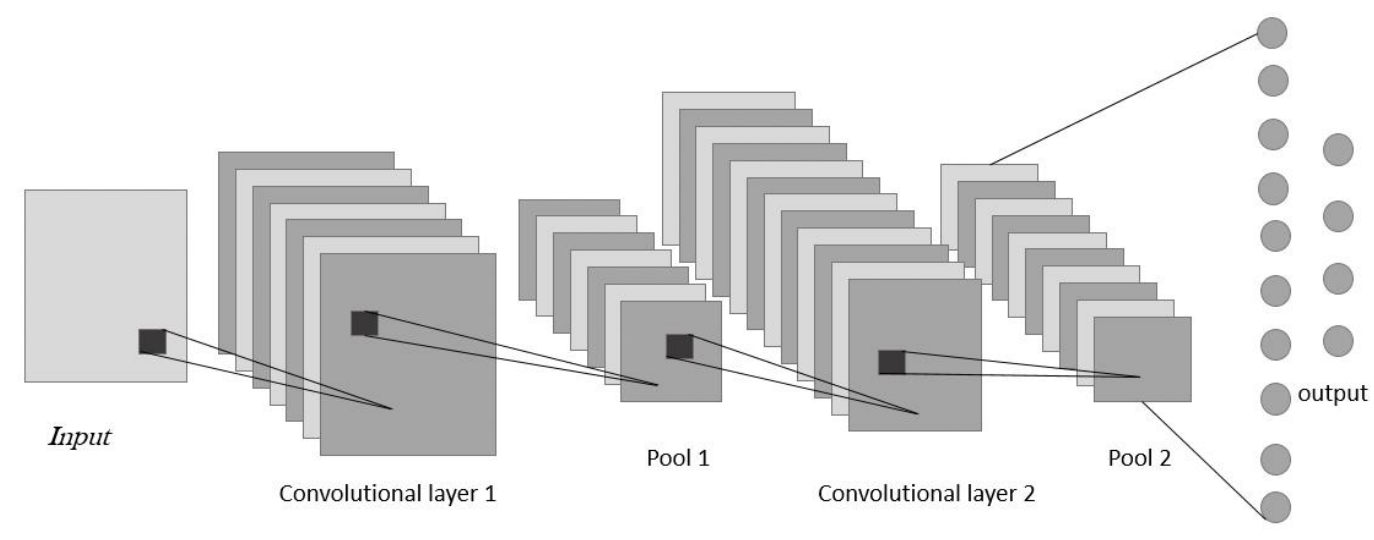

Figure 7. Convolutional network structure

The architecture of the proposed CNN is shown in Table 1. In every single layer of the CNN produces a response for the input image. In the $\mathrm{CNN}$, there are a few suitable layers for image feature extraction process. The first layers of its structure capture only the global features of the image, such as the edges and the blobs, see Figure 8, which shows a set of weights from the first layer.

In every single layer of the CNN produces a response for the input image. In the CNN, there are a few suitable layers for image feature extraction process. The first layers of its structure capture only the global features of the image, such as the edges and the blobs, see Figure 8, which shows a set of weights from the first layer. These features will be processed using deeper networks for more detailed features. After having a trained model, the evaluation process is done using the test labels with the predicted labels to find the classifier performance and accuracy. After train the segmented patches from the superpixel process, the evaluation process should be applied by repeating the same steps on unknown image to classify it and find the accuracy of the proposed system.

Table 1. Convolutional network parameters

\begin{tabular}{cc}
\hline Layer & Parameter \\
\hline Image Input 'data' & $256 \times 256$ (normalized) \\
Convolution 'conv1', & $9611 \times 11 \times 3$ convolutions \\
Max Pooling 'pool1' & $3 \times 3$ max pooling \\
Convolution 'conv2' & $2565 \times 5 \times 48$ convolutions \\
Max Pooling 'pool2' & $2 \times 2$ max pooling \\
Fully Connected 'fc6' & 4096 fully connected \\
Dropout 'drop7' & $50 \%$ \\
Classification 'output' & 2 \\
\hline
\end{tabular}

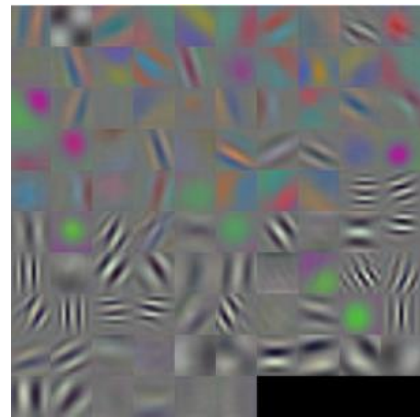

Figure 8. First convolutional layer weight 


\section{RESULTS AND DISCUSSION}

The proposed model has different training accuracy using a different number of epochs as shown in Figure 9. By using $\mathrm{CNN}$, the need for a large number of epochs is reduced where the training accuracy becomes stable, starting from 125 epoch. The system performance was evaluated, and the resulted accuracy was reported for further enhancement in the future. The overall testing accuracy was $75 \%$, and the accuracy for each class is shown in the Figure 10.

The proposed method tried to merge deep learning with the clustering for robustness purposes. These results could be improved by parameter tuning, optimisation, and apply on another type of models such as the decision tree classifier by classifying each patch individually then take the most redundant category by voting from all the image partitions. This work could be extended for multiclass classification using SVM classifier. The classification process could cover more brain tumour types by extracting more features based on machine learning.

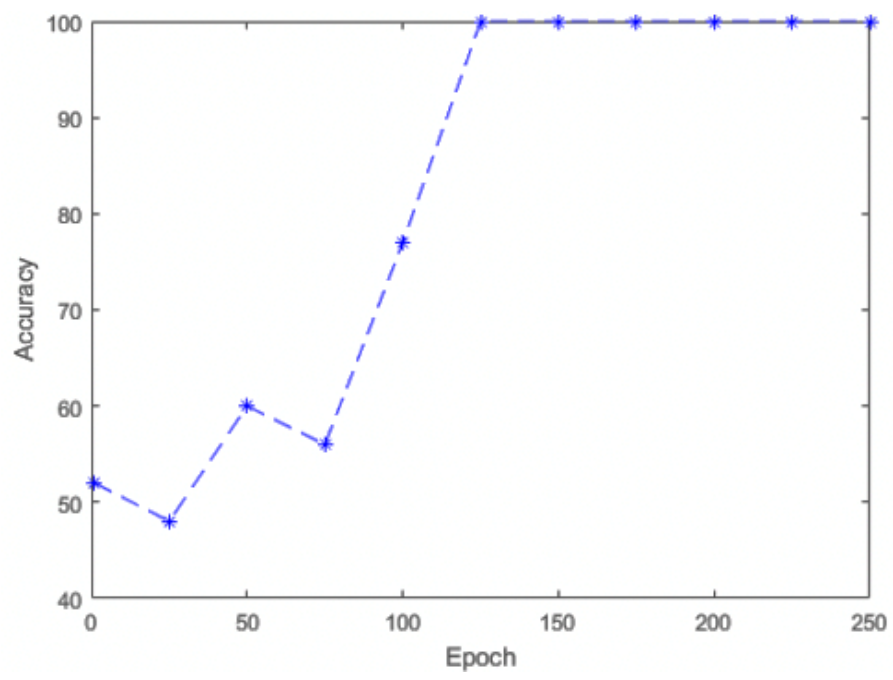

Figure 9. Training accuracy

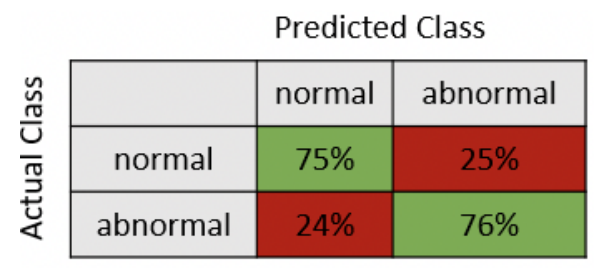

Figure 10. Confusion matrix

\section{CONCLUSION}

The brain cancer rate rises recently, which lead the research to find a high-throughput detection system. In this study, an automatic segmentation, detection, and classification system were proposed to detect the abnormal cells and identify its type. The proposed approach aims to find a robust segmentation process besides using the deep learning algorithm, which is the CNN. The segmentation using superpixel shows an effective way to segment the brain tumour cells and by using the patches which specify the image features. Using the CNN after the segmentation step abridges the feature extraction step, which is a big challenge for the researchers in machine learning algorithms. This system can be extended to cover other types of brain cancer. This system can be applied using a different number of the superpixel patches.

\section{ACKNOWLEDGEMENTS}

This research has been carried out during sabbatical leave granted to the author Moh'd Rasoul Al-Hadidi from Al-Balqa Applied University (BAU), Salt, Jordan during the academic year 2017/2018. 


\section{REFERENCES}

[1] R. L. Siegel, et al. "Cancer statistics, 2016," CA: a cancer journal for clinicians, vol. 66, no. 1, pp. 7-30, 2016.

[2] [Online], Available: http://www.khcc.jo/section/brain-tumors-0, [Retrieved 10-December-2017].

[3] M. Havaei, et al., "Brain tumour segmentation with Deep Neural Networks," Medical Image Analysis, vol. 35. pp. 18-31, 2017.

[4] "Brain Tumor Symptoms, Treatment, Support, Research," American Brain Tumor Association, [Online]. Available: www.abta.org. [Retrieved 15-December-2017].

[5] "Facts and Stats," Cure Brain Cancer Foundation, [Online]. Available: https://www.curebraincancer.org.au/page/8/facts-stats. [Retrieved 20-November-2017].

[6] M. R. Al-Hadidi, A. Alarabeyyat, and M. Alhanahnah, "Breast cancer detection using k-nearest neighbor machine learning algorithm," in 2016 9th International Conference on Developments in eSystems Engineering (DeSE), pp. 35-39, 2016.

[7] P. Hagmann, et al., "Understanding diffusion MR imaging techniques: from scalar diffusion-weighted imaging to diffusion tensor imaging and beyond," Radiographics, vol. 26, sup. 11, pp. S205 - S223, 2006.

[8] H. Hooda, O. P. Verma, and T. Singhal, "Brain tumour segmentation: A performance analysis using K-Means, Fuzzy C-Means and Region growing algorithm," in Advanced Communication Control and Computing Technologies (ICACCCT), 2014 International Conference, pp. 1621-1626, 2014.

[9] N. Sauwen, et al., "Hierarchical nonnegative matrix factorization to characterize brain tumour heterogeneity using multiparametric MRI," NMR in Biomedicine, vol. 28, no. 12, pp. 1599-1624, 2015.

[10] R. Achanta, et al. "SLIC superpixels compared to state-of-the-art superpixel methods," IEEE transactions on pattern analysis and machine intelligence, vol. 34, no. 11, pp. 2274-2282, 2012.

[11] B. AlSaaidah, et al., "Zebrafish Larvae Classification based on Decision Tree Model: A Comparative Analysis," Advances in Science, Technology and Engineering Systems Journal, vol. 3, no. 4, pp. 347-353, 2018.

[12] O. Ronneberger, P. Fischer, and T. Brox, "U-net: Convolutional networks for biomedical image segmentation," in International Conference on Medical Image Computing and Computer-Assisted Intervention, pp. 234-241, 2015.

[13] T. Kalaiselvi and P. Nagaraja, "A rapid automatic brain tumour detection method for MRI images using modified minimum error thresholding technique," Int. J. Imaging Systems and Technology, vol. 25, no. 1, pp. 77-85, 2015.

[14] D. Cobzas, et al., "3D variational brain tumour segmentation using a high dimensional feature set," in 2007 IEEE 11th International Conference on Computer Vision, pp. 1-8, 2007.

[15] M. R. Al-Hadidi, M. Y. Al-Gawagzeh, and B. A. Alsaaidah, "Solving mammography problems of breast cancer detection using artificial neural networks and image processing techniques," Indian journal of science and technology, vol. 5, no. 4, pp. 2520-2528, 2012.

[16] K. Popuri, et al., "3D variational brain tumour segmentation using Dirichlet priors on a clustered feature set," International journal of computer assisted radiology and surgery, vol. 7, no. 4, pp. 493-506, 2012.

[17] A. Krizhevsky, I. Sutskever, and G. E. Hinton, "Imagenet classification with deep convolutional neural networks," Advances in neural information processing systems, pp. 1097-1105, 2012.

[18] J. Donahue, et al. "Decaf: A deep convolutional activation feature for generic visual recognition," in International conference on machine learning, pp. 647-655, 2014.

[19] M. Jafari and S. Kasaei, "Automatic brain tissue detection in MRI images using seeded region growing segmentation and neural network classification," Australian Journal of Basic and Applied Sciences, vol. 5, no. 8, pp. 1066-1079, 2011.

[20] E. Torti, et al., "The HELICoiD project: parallel SVM for brain cancer classification," in Digital System Design (DSD), 2017 Euromicro Conference, pp. 445-450, 2017.

[21] V. Panca and Z. Rustam, "Application of machine learning on brain cancer multiclass classification," in AIP Conference Proceedings, AIP Publishing, vol. 1862, no. 1, pp. 030133, 2017.

[22] S. Jain, "Brain cancer classification using GLCM based feature extraction in artificial neural network," Int J Comput Sci Eng Technol, vol. 4, no. 7, pp. 966-970, 2013.

[23] J. J. Corso, et al., "Efficient multilevel brain tumour segmentation with integrated Bayesian model classification," IEEE transactions on medical imaging, vol. 27, no. 5, pp. 629-640, 2008.

[24] M. R. Al-Hadidi, D. Al-Hadidi, and R. S. Razouq, "Pneumonia Identification using Organizing Map Algorithm," APRN Journal of Engineering and Applied Sciences, vol. 11, no. 5, pp. 1819-6608, 2016.

[25] L. Scarpace, et al., "Data From REMBRANDT - The Cancer Imaging Archive," 2015. [Online], Available: http://doi.org/10.7937/K9/TCIA.2015.588OZUZB

[26] K.. Clark, et al., "The Cancer Imaging Archive (TCIA): Maintaining and Operating a Public Information Repository," Journal of Digital Imaging, vol. 26, no. 6, pp 1045-1057, 2013.

[27] E. Peli, "Contrast in complex images," JOSA A, vol. 7, no. 10, pp. 2032-2040, 1990.

[28] P. Neubert and P. Protzel, "Superpixel benchmark and comparison," in Proc. Forum Bildverarbeitung, vol. 6, pp. 1-12, 2012.

[29] C. A. Ortiz Toro, et al., "Superpixel-based roughness measure for multispectral satellite image segmentation," Remote sensing, vol. 7, no. 11, pp. 14620-14645, 2015.

[30] W. B. Park, et al., "Classification of crystal structure using a convolutional neural network," IUCrJ, vol. 4, no. $4,2017$.

[31] N. Tajbakhsh and K. Suzuki., "Comparing two classes of end-to-end machine-learning models in lung nodule detection and classification: MTANNs vs CNN," Pattern Recognition, vol. 63, pp. 476-486, 2017. 